\title{
A quantitative risk assessment for the occurrence of campylobacter in chickens at the point of slaughter
}

\author{
E. HARTNETT ${ }^{1}$, L. KELLY $^{1 *}$, D. NEWELL ${ }^{2}$, M. WOOLDRIDGE ${ }^{1}$ \\ AND G. GETTINBY ${ }^{3}$ \\ ${ }^{1}$ Department of Risk Research, Veterinary Laboratories Agency, Surrey, KT15 $3 N B$ \\ ${ }^{2}$ Department of Bacterial Diseases, Veterinary Laboratories Agency, Surrey, KT15 $3 N B$ \\ ${ }^{3}$ Department of Statistics and Modelling Science, University of Strathclyde, Glasgow, G1 1 XH
}

(Accepted 23 May 2001)

\section{SUMMARY}

A quantitative risk assessment model investigating the risk of human infection with campylobacter from the consumption of chicken meat/products is currently being formulated. Here such an approach is used to evaluate the probability that a random bird, selected at slaughter from Great Britain's national poultry flock, will be campylobacter-positive. This is determined from the probability that a flock chosen at random contains at least one colonized bird and the within-flock prevalence of such a flock at slaughter. The model indicates that the probability bird chosen at random being campylobacter-positive at slaughter is 0.53 . This probability value has associated uncertainty, the 5th percentile being 0.51 and the 95 th percentile $0 \cdot 55$. The model predicts that delaying the age at first exposure to campylobacter can have a significant impact on reducing the probability of a bird being campylobacter-positive at slaughter. However, implementation of current biosecurity methods makes this difficult to achieve.

\section{INTRODUCTION}

Campylobacter jejuni and C. coli are the most common cause of human acute enteritis in the UK. The reported number of cases in England and Wales in 1998 was 58000 [1]. This is a gross underestimate as many sufferers fail to seek medical attention and in some cases where medical advice is sought, the aetiology is not always investigated due to the selflimiting nature of the illness [2].

Epidemiological studies often associate the consumption of under-cooked chicken with campylobacter-related illness. This link is well-documented [3] and in one particular case-control study it has been estimated that chicken products are responsible for at least $48 \%$ of cases [4]. Such findings have led to

* Author for correspondence: Department of Risk Research, Veterinary Laboratories Agency, Woodham Lane, Addlestone, Surrey KT15 3NB. several epidemiological studies aimed at preventing campylobacter colonization of poultry [5-7], however further methods of investigating this problem are still necessary. All stages in the production of poultry are significant with regards to the contamination of the final chicken products with campylobacter. Consequently, an investigatory process involving the total supply chain, is required to facilitate the design of effective prevention and control strategies. This may be achieved through the use of quantitative risk assessment (QRA) modelling.

QRA modelling is a systematic process for describing and quantifying the risks associated with hazardous substances, processes, actions or events. The outcome is normally a mathematical statement that describes the chance of an adverse outcome from exposure to a risk at some defined level [8]. These techniques have been widely used for several years, for example in finance, however the application of such 
methods to microbial food-safety problems is relatively recent. QRA models for several microorganisms in a variety of food products have been reported. These have included Salmonella enteritidis in pasteurized liquid eggs [9], Escherichia coli O157: $\mathrm{H} 7$ in ground beef hamburgers [10] and E. coli O157:H7 in beef and beef products [11]. In addition, two farm-to-fork risk assessments have been reported by the United States Department of Agriculture (USDA) to investigate the risk of contamination of different food products with E. coli [12] and salmonella [13]. To date, no such models have been developed to estimate human health risks from campylobacter within Great Britain (GB). Such a model would identify critical control points throughout the production process. Moreover, the model development process will highlight areas of data deficiency.

This paper considers the first module of such a risk assessment, the rearing module. The model presented here estimates the probability of a random bird from within the British chicken flock being campylobacterpositive at the time of slaughter, together with an estimation of the uncertainty in this estimate. Results from this model are presented and used to demonstrate the way in which the QRA methodology can be used to obtain a better understanding of the infection pathway.

\section{METHODS}

\section{Model development}

The aim of the rearing module is to estimate the probability that a random bird from the GB poultry flock will be campylobacter-positive at the point of slaughter. This probability is defined as $P_{\mathrm{pb}}$ and can be estimated as shown in equation (1)

$P_{\mathrm{pb}}=P_{\mathrm{fp}} \times P_{\mathrm{wfp}}$,

where $P_{\mathrm{fp}}$ is the flock prevalence, that is the proportion of the national flock that is positive, and $P_{\text {wip }}$ is the within-flock prevalence of a positive flock at the time of slaughter. We define a positive flock as a flock that contains one or more birds colonized with campylobacter. Estimation of $P_{\mathrm{fp}}$ and $P_{\mathrm{wfp}}$ was undertaken as follows.

\section{Estimating flock prevalence, $\boldsymbol{P}_{\mathrm{fp}}$}

The frequent colonization of poultry flocks with campylobacter is well documented $[14,15]$ however little data exists on the prevalence of positive flocks within GB or, indeed, worldwide. Currently there are no national surveillance schemes in GB. Although some poultry production companies carry out some routine monitoring the asymptomatic nature of the colonization gives this a low priority. Consequently this highlights an area of limited data.

Sample data obtained from two fully integrated poultry companies, an epidemiological study [6] and a published source [16] were used to obtain an estimate of $P_{\mathrm{fp}}$. More specifically, individual estimates of flock prevalence were derived for each source using Beta distributions as follows

$P 1_{\mathrm{fp}}=\operatorname{Beta}\left(r_{1}+1, s_{1}-r_{1}+1\right)$,

$P 2_{\mathrm{fp}}=\operatorname{Beta}\left(r_{2}+1, s_{2}-r_{2}+1\right)$,

$P 3_{\mathrm{fp}}=\operatorname{Beta}\left(r_{3}+1, s_{3}-r_{3}+1\right)$,

$P 4_{\mathrm{fp}}=\operatorname{Beta}\left(r_{4}+1, s_{4}-r_{4}+1\right)$,

where $P 1_{\mathrm{fp}}$ and $P 2_{\mathrm{fp}}$ are estimates of flock prevalence derived from data from the two leading poultry producers. These companies together account for $35 \%$ of national chicken production. $P 3_{\mathrm{fp}}$ is an estimate of flock prevalence based on the epidemiological study [6] which involved five separate poultry producers, together responsible for $50 \%$ of the national flock, and $P 4_{f p}$ estimates flock prevalence from a published study [16]. In each case $r$ denotes the number of positive flocks and $s$ the number of flocks sampled. The Beta distribution is used to characterize the uncertainty in the sample data and assumes a random sample and that the sample size is smaller than the total population. It also assumes that each positive flock is equally likely to be detected [17]. The values corresponding to each $r$ and $s$ cannot be shown due to data confidentiality, however the resulting Beta distributions, $P 1_{\mathrm{fp}}, P 2_{\mathrm{fp}}, P 3_{\mathrm{fp}}$, and $P 4_{\mathrm{fp}}$ are shown in Figure 1.

The prevalence of positive flocks based on each source, $P 1_{\mathrm{fp}}, P 2_{\mathrm{fp}}, P 3_{\mathrm{fp}}$ and $P 4_{\mathrm{fp}}$ are weighted according to market share to give the overall flock prevalence, that is

$P_{\mathrm{fp}}=\left(P 1_{\mathrm{fp}} w 1\right)+\left(P 2_{\mathrm{fp}} w 2\right)+\left(P 3_{\mathrm{fp}} w 3\right)+\left(P 4_{\mathrm{fp}} w 4\right)$,

where $w 1, w 2, w 3$ and $w 4$ are the associated weights. The values for $w 1, w 2, w 3$ and $w 4$ are based on the companies' market share using denominator data derived from MAFF statistics [18].

\section{Estimating within-flock prevalence, $\boldsymbol{P}_{\text {wfp }}$}

Within-flock prevalence (WFP) is a measure based on the number of birds expected to be colonized with campylobacter within a positive flock. The WFP is 


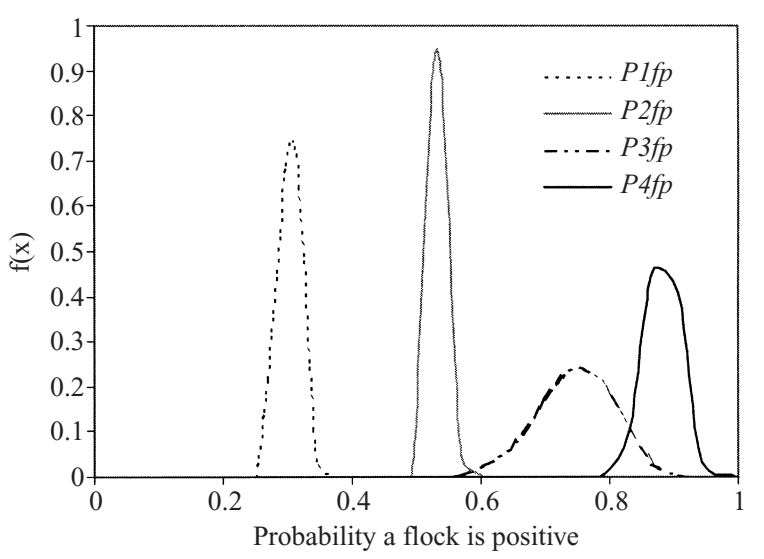

Fig. 1. Graph to show the uncertainty distributions for the probability that a flock is positive for each of the data sources $P 1_{\mathrm{fp}}, P 2_{\mathrm{fp}}, P 3_{\mathrm{fp}}$, and $P 4_{\mathrm{fp}}$.

directly related to the rate of transmission and is therefore a time-dependent phenomenon for a positive flock. It has been reported that the within-flock transmission of campylobacter is rapid and that once campylobacter has been detected the WFP reaches $100 \%$ within 7 days $[7,19]$. However the dynamics of campylobacter transmission in poultry flocks is poorly understood.

Mathematical models have been previously used to investigate the pattern of disease epidemics $[20,21]$ in both human and animal populations. Here, we adopt a mathematical approach to describe the transmission of campylobacter within a flock.

Poultry production is highly specialized and follows a defined structure [22]. Briefly, when the birds are 1 day old they are taken to a broiler-growing farm, where they remain until they reach slaughter weight at ages between 30 and 60 days to become 'table birds'. At this point depopulation occurs; that is birds are removed from the house, and transported to the slaughter facility for processing to produce the sale product.

Upon arrival at the growing farms the birds are placed in a house where they form spatial clusters. This clustering effect is likely to be due to social factors. The display of social behaviour is common to fowl and has been well documented [23-28] and experimental work suggests a similar social behaviour is displayed by birds in the commercial rearing environment [29]. The area explored by a given bird diminishes with age [29] thus enhancing the clustering effect. This reduction can be attributed to the increase in size of birds in a fixed environment.

The transmission of campylobacter in a flock is believed to begin with a single bird becoming colonized. The mechanism by which a single bird becomes colonized and the time at which this occurs is unknown. Case-control studies and typing schemes have demonstrated several reservoirs to which a flock may be exposed. These include wild birds, rodents, and cross-contamination from the environment via farm workers [30, 31]. It seems likely that transmission is initially confined to the cluster containing the firstcolonized bird. During this process campylobacters are excreted in the faeces of positive birds. This results in the contamination of the feed and water. Typically a threshold will be reached where the contamination level is sufficient to cause extensive colonization in birds as a result of the ingestion of contaminated feed or water. This allows for dissemination of campylobacters throughout the whole flock until all birds are colonized.

It is therefore appropriate to model the process of flock colonization in two stages. The first stage is the initial transmission within the cluster containing the first bird that is colonized, and the second stage is the transmission throughout the remainder of the flock.

Within this model it is assumed that the first bird becomes colonized at a time denoted $t_{\mathrm{ex}}$. This time is defined as the age at first successful exposure of a bird in the flock, that is exposure which leads to colonization with campylobacter. This time is set to $t=t_{0}$. Stage 1 is described by a modified chain binomial model until a threshold time is reached. Experimental studies have shown that, following colonization of the first bird, campylobacters can be detected in the feed, water and litter after 3 days [19]. It is therefore assumed that the levels of contamination become sufficient to allow widespread dissemination of the organism throughout the flock 4 days following colonization of the first bird. Thus a model for simple epidemic spread can be used to represent the second stage of the colonization process. Thereafter transmission continues until either all birds become colonized or depopulation occurs at time $t_{A}$. Each of these stages can be described by the following models.

\section{Stage 1: Chain binomial}

The initial transmission is described using a chainbinomial model of epidemic spread [20, 32]. Such a model is deemed appropriate when the data available for parameter estimation are measured in discrete time [20] as in the occurrence of colonized birds within the cluster containing the first positive bird.

The basic chain binomial model describes the 
colonization of a random susceptible bird which becomes colonized after a fixed constant time. The colonized bird is then removed from the susceptible population. New cases occur within the cluster in distinct groups at each time point, as described by the recurrence equation (2)

$I_{\mathrm{c}}(t+1)=I_{\mathrm{c}}(t)+N I_{\mathrm{c}}(t+1)$,

where $I_{\mathrm{c}}(t)$ is the number of colonized birds in the cluster at $t$, and $N I_{\mathrm{c}}(t+1)$ is the number of newly colonized birds in the period $(t, t+1]$ when $(t, t+1]$ is defined as 1 day. The number of newly colonized birds at each time-point will follow a binomial distribution which depends upon the probability that a susceptible bird in the cluster becomes infected in time $(t, t+1]$, that is $p(\mathrm{t})$. Following on from this, the binomial likelihood for $N I_{\mathrm{c}}(t+1)$ can be written as:

$$
\begin{aligned}
& P\left[N I_{\mathrm{c}}(t+1)=x_{t+1}, N I_{\mathrm{c}}(t)=x_{t}, \ldots, N I_{\mathrm{c}}(1)\right. \\
& \left.\quad=x_{i} \mid I_{\mathrm{c}}(0)=x_{0}\right]=\Pi_{i} P\left[N I_{\mathrm{c}}(i)=x_{i} \mid H(i-1)\right],
\end{aligned}
$$

where this binomial likelihood is given by the binomial probabilities dependent on $p(t)$, the probability that a susceptible bird becomes colonized in the period $(t$, $t+1$ ], and $H(t)$ can be described as the history of the epidemic up to that point. More specifically

$$
\begin{aligned}
& P\left[N I_{\mathrm{c}}(t+1)=x_{t+1} \mid H(t)\right] \\
& =\left(\begin{array}{c}
S_{\mathrm{c}}(t) \\
x_{t+1}
\end{array}\right) p(t)^{x_{t+1}[1-p(t)]^{S_{\mathrm{c}}(t)-x_{t+1}},} \\
& \begin{aligned}
H(t)=\left\{N I_{\mathrm{c}}(t)=x_{t}, N I_{\mathrm{c}}(t-1)=x_{t-1}, \ldots, N I_{\mathrm{c}}(1)\right. \\
\left.=x_{1}, I(0)=x_{0}\right\},
\end{aligned}
\end{aligned}
$$

where $S_{\mathrm{c}}(\mathrm{t})$ is the number of susceptible birds in the cluster at time $t$.

When considering transmission of campylobacter within a flock, the probability that a bird becomes colonized is dependent upon the transmission rate, the social need to make contact with other birds, and the probability of contact with a colonized bird. The model assumes a randomly mixing population, that is a given bird is equally likely to make a contact with every infected bird [32]. However commercial flocks can be many thousands in size hence random mixing is not a reasonable assumption. By assuming a bird moves around a limited number of birds, a cluster, and considering the number of birds a given bird comes into contact with, and the number of times contact is made we are able to model the spread of infection in a small neighbourhood. The basic chain binomial model described above is modified to include these factors. The use of the modified chain binomial model makes several assumptions [33]: (i) the total cluster size remains constant, i.e. $S_{\mathrm{c}}(t)+I_{\mathrm{c}}(t)=n_{\mathrm{c}}$ for all values of $t$ where $n_{\mathrm{c}}$ is the total cluster size;

(ii) a bird, which becomes colonized at time $t$, cannot transmit the organism to another bird until time $t+1$, this allows for the fixed latent period of 1 day;

(iii) birds within the cluster act independently;

(iv) each non-colonized bird has the same probability of being colonized at time $t$.

Let $b$ equal the probability of transmission given a single contact of a susceptible bird with a colonized bird, $A$ equal the number of birds a given bird comes into contact with in 1 day, that is $(t, t+1]$ and $R$ equal the number of times the bird is contacted by each of the $A$ contacts in $(t, t+1]$, where $A$ and $R$ are random variables which have probability density functions given by

$P(A=a)=f(a)$,

$P(R=r)=g(r)$.

Probability generating functions are used for $A$ and $R$ as they are easier to manipulate [32]. The associated probability generating functions are given by

$\Phi_{A}(s)=E\left(s^{A}\right)=\sum_{a=0}^{\infty} f(a) s^{a}$,

$\Phi_{R}(s)=E\left(s^{R}\right)=\sum_{r=0}^{\infty} g(r) s^{r} . \quad(0 \leqslant s \leqslant 1)$

From the work of $\mathrm{Ng}$ and Orav [33], assuming independence of individual birds, the probability that a susceptible bird becomes colonized in the period $(t$, $t+1], p(t)$, is given by equation (3)

$p(t)=1-\Phi_{A}\left[1-\left\{\left[\frac{I_{\mathrm{c}}(t)}{n_{\mathrm{c}}(t)}\right]\left[1-\Phi_{R}(1-b)\right]\right\}\right]$.

This can be written equivalently:

$p(t)=1-\sum_{a} f(a)\left\{1-\frac{I_{\mathrm{c}}(t)}{n_{\mathrm{c}}(t)}\left[1-\sum_{r} g(r)(1-b)^{r}\right]\right\}^{a}$.

It is assumed that the number of contacts with an individual in 1 day follows a binomial distribution, i.e. $\operatorname{Binomial}\left(n_{\mathrm{c}} P_{\mathrm{c}}\right)$ and the number of times that a bird makes contact with a given bird follows a Poisson distribution, i.e. Poisson $(y)$ where $P_{\mathrm{c}}$ is the probability that contact is made with another bird, and $y$ is the mean number of times contact is made with each bird. In this way the number of contacts is limited to be equal to or less than the cluster size, but the number of times contact is made is theoretically unbounded. The generating functions for the number 


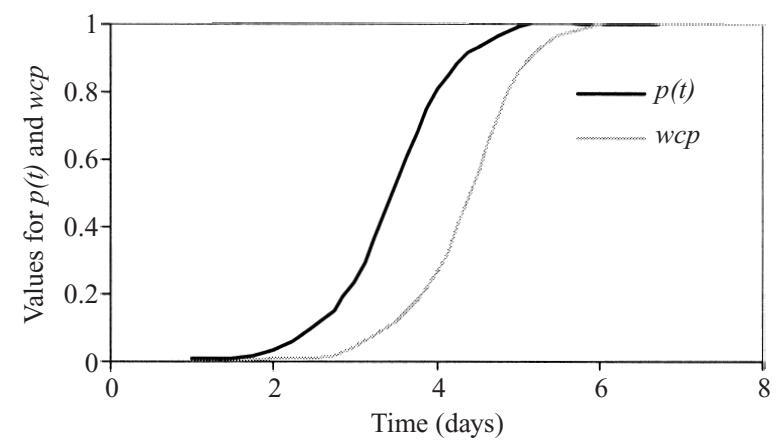

Fig. 2. Graph to show how the probability that a noncolonized bird will become colonized, $p(t)$, and the resultant prevalence of campylobacter-positive birds within a cluster, $w c p$, for a given cluster vary over time. Here the mean number of times contact is made with each bird is five contacts per bird per day, the number of possible contacts is 109 contacts per day and the probability that contact is made is 0.07 .

of contacts made, $\Phi_{A}$ and the number of times contact is made with each bird, $\Phi_{R}$ are therefore given by

$\Phi_{A}=\left(1-P_{\mathrm{c}}+P_{\mathrm{c}} s\right)^{n_{\mathrm{c}}}$, $\Phi_{R}=\mathrm{e}^{(-y(1-s))}$.

Thus substituting these generating functions into equation (3), the probability that a non-colonized bird becomes colonized in 1 day, that is $p(t)$, is given by:

$p(t)=1-\left[1-P_{\mathrm{c}}\left(\frac{I_{\mathrm{c}}(t)}{n_{\mathrm{c}}(t)}\right)\left(\frac{1-\exp ^{-y b}}{1-\exp ^{-y}}\right)\right]^{n_{\mathrm{c}}}$.

The mean number of newly colonized birds is then given by:

$N I_{\mathrm{c}}(t+1)=p(t) S_{\mathrm{c}}(t)$.

The way in which $p(t)$ and the resultant prevalence of positive birds within a given cluster varies over time is illustrated in Figure 2 where $y$, the mean number of times contact is made with another bird is five contacts per bird per day, $n_{\mathrm{c}}$ the number of possible contacts is 109 contacts per day, where 109 is the mean value of the distribution, and $P_{\mathrm{c}}$ the probability that contact is made is 0.07 . It can be seen that as the prevalence within a cluster increases so does $p(t)$. Further, the probability that a bird becomes colonized is greater than the prevalence of positive birds in the cluster. This is due to the occurrence of multiple contacts per bird with another bird.

\section{Stage 2: Epidemic spread}

As previously discussed a threshold time is reached when the water and feed become contaminated with campylobacter, this threshold normally occurs 4 days after the first bird in the cluster becomes colonized. Thereafter stage 2 begins at time $t=t_{5}$. In the second stage the number of newly colonized birds at any time-point is dependent upon the initial number of colonized birds, that is the number of birds colonized within the cluster, modelled in stage 1 , at the time when stage 2 begins which is given by $I_{\mathrm{c}}\left(t_{4}\right)$ and the transmission rate. Therefore the colonization process in stage 2 can be represented by the use of a simple epidemic model. It is assumed that in stage 2 there is a flock of size $n$. The colonization process in stage 2 begins with $I_{\mathrm{c}}\left(t_{4}\right)$ colonized birds and $S_{B}\left(t_{4}\right)$ susceptible birds, that is the number of colonized birds at the end of 4 days after the first bird in the cluster became colonized, where

$S_{B}\left(t_{4}\right)=n-I_{\mathrm{c}}\left(t_{4}\right)$.

The number of newly colonized birds is proportional to both the numbers of colonized and susceptible birds. Therefore the process can be described by the differential equation (4)

$\frac{\mathrm{d} S_{B}}{\mathrm{~d} t^{\prime}}=-b_{B} S_{B}\left(t^{\prime}\right)\left[n-S_{B}\left(t^{\prime}\right)\right]$,

where $S_{B}(t)$ is the number of susceptible birds in the second stage, $b_{B}$ is the biological transmission probability adjusted to allow for the large flock sizes and when $t^{\prime}$ is equal to $(t-4)$ where the value 4 is the time in days until the second stage begins. By incorporating $t^{\prime}$ into the differential equation the result is a small lag in the epidemic curve at the point when the change occurs from the first to the second stages of the model. This is biologically consistent as the organism changes mode of transmission, from bird to bird to environmental transmission from feed and water. The transmission probability, $b_{B}$ is proportional to the transmission probability $b$. Transmission in the second stage is not exclusively via bird-to-bird contact, but also includes feed and water. However transmission in the second stage is indirect bird-to-bird contact as it is the colonized birds which contaminate the feed and water and the level of contamination depends on the number of colonized birds.

Solving (4) for the number of susceptibles gives equation (5).

$S_{B}\left(t^{\prime}\right)=\frac{S_{B}\left(t_{4}\right) n}{S_{B}\left(t_{4}\right)+I_{\mathrm{c}}\left(t_{4}\right) \exp ^{\left[n b_{B} t^{\prime}\right]}}$.

After completion of the first and second stages the 


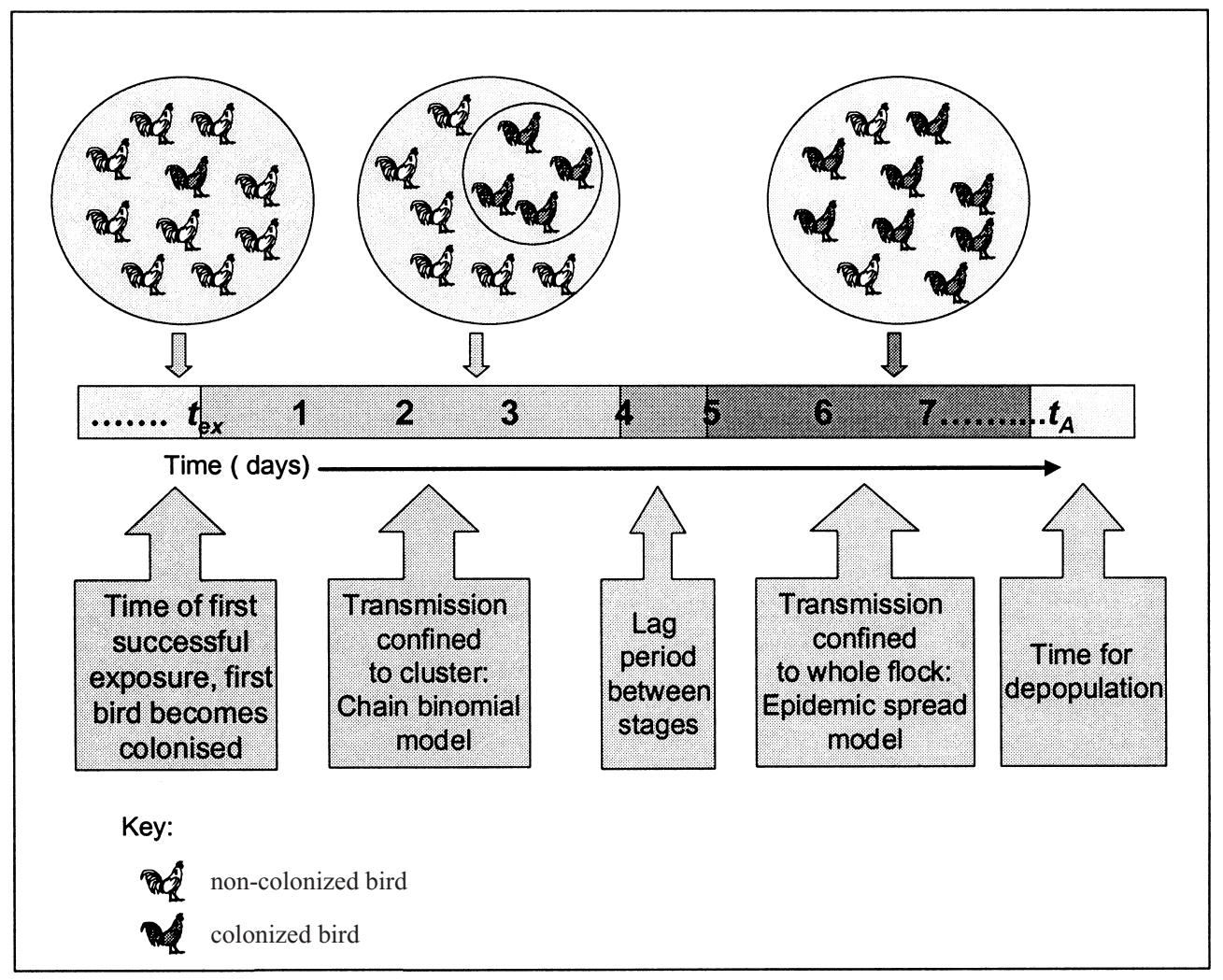

Fig. 3. Schematic representation of the model to estimate the within-flock prevalence of a campylobacter-positive flock at slaughter, $P_{\text {wfp }}$.

total number of colonized birds within a flock $I(t)$ is given by

$I(t)=n-S_{B}(t)$.

Therefore the within-flock prevalence at time $t$ since the time of exposure can be calculated directly from equation (6):

$P_{\mathrm{wfp}}(t)=\frac{I(t)}{n}$.

A schematic representation of the overall model used to estimate the within-flock prevalence of a positive flock at slaughter, $P_{\text {wfp }}$, is shown in Figure 3.

\section{Parameter estimation and simulation}

The model presented here is required to be stochastic to account for real-life variability and uncertainty. Probability distributions are derived for input variables using available data and, where necessary, expert opinion. These distributions are then incorporated within a Monte-Carlo simulation and output estimates of risk generated. These output values can similarly be described in terms of probability distributions and consequently required risks can be summarized in terms of mean values, associated confidence intervals, etc. In this way the uncertainty concerning outputs can be evaluated.

The parameters and their estimated distributions are listed in Table 1. There is extensive published work on campylobacter, however the number of studies that investigate the dynamics of within-flock transmission of this organism is limited. As a result values for $A, R$, and $n_{\mathrm{c}}$ are based upon expert opinion. Experts, including a veterinary epidemiologist, avian ecologist and broiler farm manager, selected for their experience with broiler flocks were asked to provide estimates for minimum, most-likely and maximum values for $A, R$, and $n_{\mathrm{c}}$. These estimates have been used to define triangular distributions and opinions are combined within a discrete distribution as described in Vose [17]. More specifically, by using Discrete $\left(\left\{E_{1}, E_{2}, \ldots, E_{n}\right\},\left\{w_{E 1}, w_{E 2}, \ldots, w_{E n}\right\}\right)$, where $E_{1}, E_{2}, \ldots, E_{n}$ are $n$ individual experts opinions, defined by the associated triangular distributions, and $w_{E 1}, w_{E 2}, \ldots, w_{E n}$ are the associated weights of each opinions. Each expert is given equal weighting.

The biological transmission rate for campylobacter, $b$, is based upon experimental studies $[19,34]$. These studies involved the placing of a colonized bird in a 
Table 1. Probability distributions and associated parameter values used in the QRA model to estimate the probability distribution for a random bird selected from the UK chicken flock being campylobacter-positive at the point of slaughter

\begin{tabular}{|c|c|c|}
\hline Parameter & Symbol & $\begin{array}{l}\text { Probability } \\
\text { representation }\end{array}$ \\
\hline \multicolumn{3}{|l|}{ Experimental data } \\
\hline Transmission rate per day & $b$ & Uniform $(0 \cdot 1,0 \cdot 3)$ \\
\hline \multicolumn{3}{|l|}{ Expert opinion } \\
\hline $\begin{array}{l}\text { Number of contacts a bird makes } \\
\text { with other birds in } 1 \text { day }\end{array}$ & $A$ & $\begin{aligned} & \text { Risk Discrete }\left(\{\alpha, \beta, \gamma\},\left\{P_{\alpha}, P_{\beta}, P_{\gamma}\right\}\right) \\
& \text { Where: } \alpha \sim \text { Triang }(12,100,500)^{*} \\
& \beta \sim \text { Triang }(30,50,120)^{*} \\
& \gamma \sim \text { Triang }(20,45,100)^{*}\end{aligned}$ \\
\hline $\begin{array}{l}\text { Number of times a bird comes into } \\
\text { contact with a given bird in } 1 \text { day }\end{array}$ & $R$ & $\begin{aligned} & \text { RiskDiscrete }\left(\{\alpha, \sigma\},\left\{P_{\lambda}, P_{\sigma}\right\}\right) \\
& \text { Where: } \lambda \sim \text { Triang }(3,5,6)^{*} \\
& \delta \sim \text { Triang }(2,6,8)^{*}\end{aligned}$ \\
\hline Size of cluster & $n_{\mathrm{c}}$ & $\begin{array}{l}\text { RiskDiscrete }\left(\{\mu, \omega\},\left\{P_{\mu}, P_{\omega}\right\}\right. \\
\text { Where: } \mu \sim \text { Triang }(N / 12, N / 10, N / 8)^{*} \\
\quad \omega \sim \text { Triang }(100,300,1000)^{*}\end{array}$ \\
\hline \multicolumn{3}{|l|}{ Industrial data } \\
\hline Flock size & $n$ & Triang $(7800,30750,41596)$ \\
\hline Age at depopulation in days & $t_{A}$ & Triang $(28,42,64)$ \\
\hline $\begin{array}{l}\text { Age at first exposure to campylobacter } \\
\text { in days }\end{array}$ & $t_{\mathrm{ex}}$ & Uniform $\left(14, t_{A}\right)$ \\
\hline
\end{tabular}

* These parameters are Triangular distributions based on expert estimates.

group of un-colonized birds. Samples were then taken daily to measure the change in the number of colonized birds over time. From these studies two values for transmission rate were estimated and used to define a uniform distribution; that is, all values between the two values of $b$ are assumed equally likely to be the estimated value for a given flock. Ideally, more information is required, for example the most likely value of $b$ within the range of these two values. If this information were available the use of a triangular distribution would allow values within the range to be weighted, providing a more realistic estimate for this parameter. The value of $b_{B}$ is proportional to $b$ as previously described. The proportionality factor is equal to $1 / 10 n$. Due to the absence of data, experts in the area of the colonization of chickens agreed this factor with campylobacter by inspection of the resulting epidemic curve.

The age at first successful exposure, $t_{\mathrm{ex}}$, is an unknown parameter in the model. Several studies have shown that campylobacters are rarely isolated from commercial flocks under 3 weeks of age. One explanation of this is that the colonization process probably begins with a single bird and it is possible that it takes time before positive birds are detectable in large commercial flocks. It is therefore assumed that the time until the number of birds colonized is large enough to allow detection, after exposure to campylobacters, is 1 week. Therefore, the time of exposure, $t_{\mathrm{ex}}$, is assumed to be a uniform random variable between 14 days and the age at depopulation.

Finally, distributions for $n$, and $t_{A}$ are derived directly from data involving several industrial sources that together are representative of approximately $50 \%$ of the national flock.

The simulation model was developed in the software package@Risk ( $@$ Palisade Corp.). To run the model simulations were carried out in two parts. An initial assumption is made that prior to the time at first successful exposure to campylobacter and appearance of the first positive bird, $t_{\mathrm{ex}}$ the within flock prevalence for a given flock is zero.

In the first part of the simulation the two-stage model used to calculate the within-flock prevalence, $p_{\mathrm{wfp}}$ is run. More specifically, values for $t_{\mathrm{ex}}, t_{A}$, are randomly selected from the associated distributions (shown in Table 1) and used to generate the time to run the within-flock prevalence model. This is a result of the time of first successful exposure and the age at slaughter for a given flock, and is given by $t_{\text {run }}=$ $t_{A}-t_{\mathrm{ex}}$, where $t_{A}$ is the age at slaughter, $t_{\mathrm{ex}}$ is the time of first successful exposure and $t_{\text {run }}$ is the time for which the within-flock prevalence model is run. Values for $n, b, R, A$ are then selected from the associated 


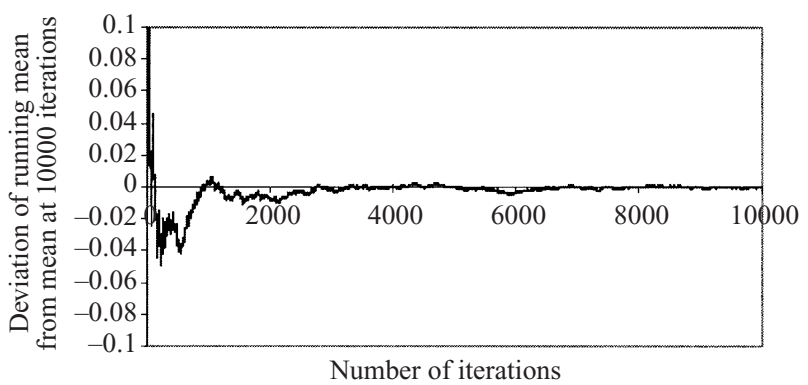

Fig. 4. Graph to show the variation in the running mean of the probability that a bird will be campylobacter-positive at slaughter, $P_{\mathrm{pb}}$, from the mean of this probability for numbers of iterations up to 10000 .

distributions and $P_{\text {wfp }}$ estimated. This process is repeated 4000 times. The result is a variability distribution for $P_{\text {wfp }}$. Therefore, for a random flock the within flock prevalence is the sum of each within flock prevalence weighted by the frequency of occurrence of that prevalence. More specifically

$P_{\mathrm{wfp}}=\sum_{i=1}^{i=4000} P_{\mathrm{wfp}_{i}} f\left(P_{\mathrm{wfp}_{i}}\right)$.

This is equivalent to taking the mean of the distribution. Following on from this, for the second part of the simulation 1000 values for $P_{\mathrm{fp}}$ are randomly selected from the distribution and $P_{\mathrm{pb}}$ is then calculated as shown in equation (1). The result is $P_{\mathrm{pb}}$ and the associated uncertainty distribution.

The number of iterations for the first simulation, that is to calculate $P_{\text {wfp }}$, was chosen according to when the model output mean was considered stable [17] that is when it varied less than $1 \%$ from the mean output at 5000 iterations. The variation from the mean at 10000 iterations for a given number of iterations is shown in Figure 4. It can be seen that the model output stabilizes at 4000 iterations. The number iterations for the second stage, calculation of $P_{\mathrm{pb}}$ was chosen to allow adequate selection of the range of values from the distribution for the flock prevalence, $P_{\mathrm{pp}}$. Values above those selected did not result in any notable differences to output estimates for the model.

\section{RESULTS AND DISCUSSION}

The cumulative and density uncertainty plots for the probability that a random bird is campylobacterpositive, $P_{\mathrm{pb}}$, using Latin Hypercube sampling, is shown in Figure 5. From the model results, although $25 \%$ of randomly selected birds from the national flock will have a probability of less than 0.52 of being
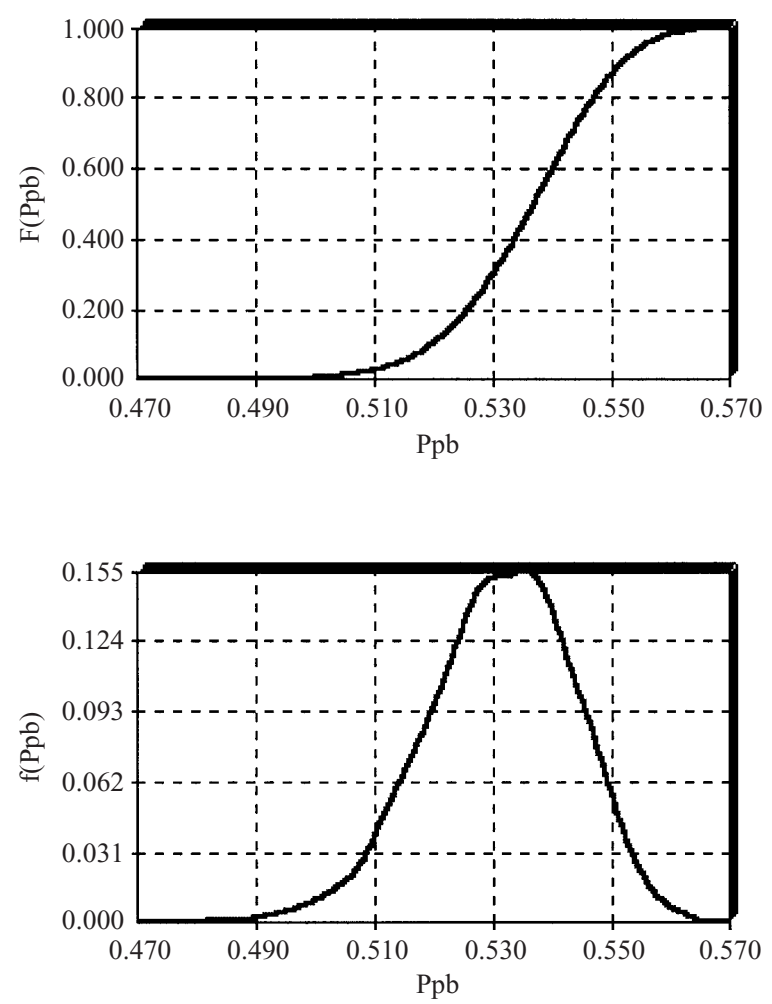

Fig. 5. Cumulative and density plots for the probability a random bird selected at slaughter from the GB chicken flock is campylobacter-positive.

campylobacter-positive, the most probable value that a bird is positive is 0.53 . Moreover, $25 \%$ of randomly selected birds from the national flock will have a probability in excess of 0.54 of being campylobacterpositive. The density plot indicates that a bird either has a high probability of being campylobacter-positive or the bird will be campylobacter-negative. It is unlikely that a bird will have a low probability of being campylobacter-positive. This can be accounted for in two ways. First, following exposure to campylobacter colonization of birds is an all-or-nothing event. Birds that are colonized will shed the organism, birds that are not colonized with campylobacter will not. Secondly, as previously discussed the transmission of campylobacter colonization within a flock is rapid, therefore once a flock has been exposed it is likely to have a within-flock prevalence approaching $100 \%$ at the point of depopulation. From equation (1) it can be seen that as the within-flock prevalence probability, $P_{\text {wfp }}$ increases, so does the probability that a random bird is positive, $P_{\mathrm{pb}}$. Hence $P_{\mathrm{pb}}$ is highly dependent on $P_{\text {wfp }}$.

The sensitivity of the probability that a random bird is campylobacter-positive, $P_{\mathrm{pb}}$, to the age at first successful exposure, $t_{\mathrm{ex}}$, the transmission rate, $b$, cluster 


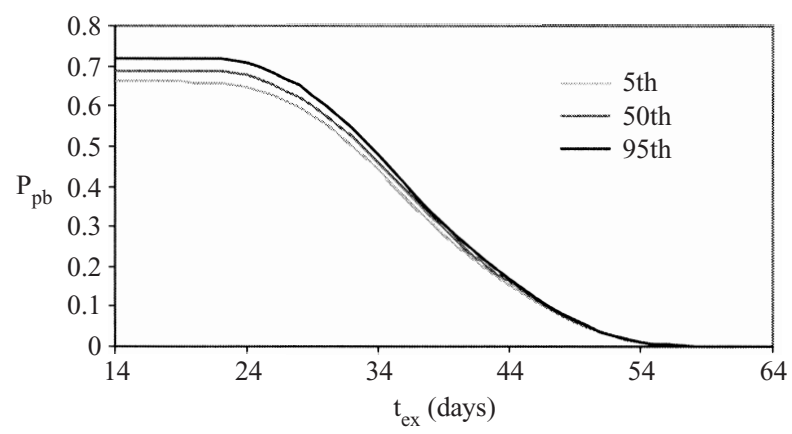

Fig. 6. The sensitivity of the probability that a random bird is campylobacter-positive $\left(P_{\mathrm{pb}}\right)$ to the time of first exposure $\left(t_{\mathrm{ex}}\right)$.

size, $n_{\mathrm{c}}$, number of contacts made in one day, $A$, and the time of depopulation, $t_{A}$, was examined. For a given variable, $t_{\mathrm{ex}}, b, n_{\mathrm{c}}, A$, and $t_{A}$, the value was varied across the minimum and maximum values of the distribution while all other parameters remained as described by their associated distributions (Table 1). This revealed that $P_{\mathrm{pb}}$ is most sensitive to the time of first successful exposure, $t_{\mathrm{ex}}$, and the time of depopulation, $t_{A}$. Variation in the other parameters investigated had minimal effects upon output estimates of $P_{\mathrm{pb}}$.

The way in which $P_{\mathrm{pb}}$ varies with $t_{\mathrm{ex}}$ is shown in Figure 6. From this it can be seen that as the age at first successful exposure $\left(t_{\mathrm{ex}}\right)$ increases the probability that a bird is campylobacter-positive can be reduced. This is a reflection of the time at which depopulation occurs. As the time till first successful exposure increases the number of days until depopulation decreases thus reducing the chance of flock colonization resulting in a diminished within flock prevalence. Similarly a reduction in the time to depopulation, $t_{A}$, also results in a reduced $P_{\mathrm{pb}}$.

The model indicates that the probability of a bird being positive at slaughter changes over time. Thus by delaying the time until first successful exposure the probability of a random bird being campylobacter positive is reduced. For example an increase in $t_{\mathrm{ex}}$ from 30 to 40 days results in a drop in the 50th percentile from 0.57 to 0.26 (Fig. 6). This reduction suggests that this is a potential critical control point. This is an intuitive result as delaying the time at which a flock becomes positive will reduce the number of birds which become colonized before the flock is removed for slaughter. Previous reports indicate that the implementation of strict biosecurity measures, such as boot dips and sanitary barriers [6, 35] can delay the time until first successful exposure. However, the successful application of such measures in the day- to-day workings of a poultry farm is difficult, with compliance by staff difficult to monitor [36]. Thus delaying the time until first successful exposure may prove an impractical strategy. An alternative is to reduce the age at depopulation that also reduces the probability a random bird is positive. Although this is also a potential critical control point, given market demands on size and weight of table birds at sale, this is not a feasible option.

Within this manuscript a model is presented which estimates the probability that a bird is campylobacterpositive at the point of slaughter based upon an estimate for the prevalence of campylobacter-positive flocks and the probable within-flock prevalence of a positive flock. It should be noted that given data on the size of individual flocks and the associated withinflock prevalence basic probability theory can be used to estimate the probability that a bird selected at random is positive at slaughter. However, it is highly unlikely that such information will be available given the large flock sizes and the cost and difficulty of sampling a large number of individual birds from these flocks. Therefore an alternative method must be developed. Such a method is described here. It can be shown that the two methods are equivalent. For example, consider the estimation of $P_{\mathrm{pb}}$ given there are $m$ flocks in total in the country. The probability $P_{\mathrm{pb}}$ is estimated as follows

$P_{\mathrm{pb}}=\sum_{i=1}^{m} P($ bird is from flock $i$ and flock $i$ is

positive and bird is positive),

where

$P$ (bird is from flock $i$ and flock $i$ is positive and bird is positive $)=P($ bird is from flock $i)$ $\times P$ (flock $i$ is positive) $\times P$ (bird positive given from flock $i$ and flock $i$ is positive).

Therefore given $n_{i}$ is the flock size of flock $i, N$ is the total population size, $\mathrm{pf}_{i}$ is the probability that flock $i$ is positive and $\mathrm{wfp}_{i}$ the within-flock prevalence of flock $i$,

$$
\begin{aligned}
P_{\mathrm{pb}}= & \left(\frac{n_{1}}{N} \times \mathrm{pf}_{1} \times \mathrm{wfp}_{1}\right)+\left(\frac{n_{2}}{N} \times \mathrm{pf}_{2} \times \mathrm{wfp}_{2}\right) \\
& +\cdots+\left(\frac{n_{m}}{N} \times \mathrm{pf}_{m} \times \mathrm{wfp}_{m}\right) .
\end{aligned}
$$

Therefore in the general case

$P_{\mathrm{pb}}=\mathrm{pf} \times \sum_{i=1}^{m} \frac{n_{i}}{N} \times \mathrm{wfp}_{i}$, 
where $\Sigma_{i=1}^{m}\left(n_{i} / N\right) \times \mathrm{wfp}_{i}$ is the mean within-flock prevalence, and hence is equivalent to equation (1).

The current model does not consider the source(s) of campylobacter colonization. Why certain flocks become campylobacter-positive and others remain campylobacter-free until slaughter is unknown. Campylobacter is a ubiquitous organism in the environment and, as such, there are many potentially contaminated sources to which a flock may be exposed. Considering this it is possible that different sources of campylobacter result in different withinflock dynamics for the colonization process. Such factors can be incorporated into the model.

The assumptions for the model that represents the process of flock colonization, that is estimation of $P_{\mathrm{wfp}}$, are important in the interpretation of the model results. Within the current model, it is assumed that a flock initially comprises of birds in clusters. Successful colonization is derived from a single bird in one cluster. The organism is then disseminated, initially by direct contact with the colonized bird and then via contaminated feed and water. This assumption may be incorrect. Campylobacters are frequently isolated from water sources and contaminated water has been associated with human outbreaks of campylobacteriosis [37]. If a flock is exposed to contaminated water multiple colonized birds will initiate the colonization process. Homogeneous mixing could then be expected as the water is circulated through the house. This could be described by use of the differential equation for epidemic spread, that is equation (4), and disregarding the chain binomial model. In addition it is debatable whether vertical transmission of campylobacters can occur [38, 39]. Certainly if vertical transmission does occur it is likely to be an infrequent event with only up to 10 out of 1000 birds being colonized via this route [8]. Such an occurrence would result in multiple colonized birds and multiple initial clusters containing colonized birds. This can be modelled by modification of the chain binomial model to sum the number of newly colonized birds in each cluster at each time point [33].

At present the frequency with which flocks are exposed to different sources of campylobacter is unknown. Once such information becomes available the incorporation of source of organism and resulting within-flock dynamics may lead to a model that more accurately represents real life. However, given the rapidity of dissemination of campylobacter through a flock it is unlikely that inclusion of source of organism would have a large impact upon current estimates for
$P_{\mathrm{pb}}$. Experimental studies have demonstrated that as campylobacter is passaged through birds the colonization potential of the organism increases [40]. The current model assumes a constant transmission rate. By allowing the transmission rate, $b$, to vary according to either time or the number of birds that are colonized, a better representation of the flock colonization process may be obtainable. Increased colonization potential would lead to a reduced time for within flock spread of the organism and as a result it may be that the distribution presented here for $P_{\mathrm{pb}}$ is an understimate of the true value. Consequently, the true number of birds that are campylobacterpositive at slaughter may be even greater than is estimated by the current model results. Certainly this would be in agreement with some poultry survey results [6].

In the absence of data, experts provided estimates of parameter values. By using expert opinion the assumption is made that the true value of the parameter in question is included in the estimates given. In this model, expert opinion was obtained for parameters $A, R$, and $n_{\mathrm{c}}$ (Table 1). It can be seen that estimates of $A$ and $R$ are consistent, however estimates on $n_{\mathrm{c}}$ would appear to be inconsistent. Inconsistency between experts is a debatable issue. If there is information that would suggest one opinion is not valid then that opinion should not be used. However, if there is information available on the parameter in question, then expert opinion would not be the method of choice to obtain parameter estimates. For a full discussion on the use of expert opinion in risk assessment modelling the reader is directed to [17].

The model considers the probability that a random bird selected from the national slaughter flock will be campylobacter-positive. The results presented here can be validated by the implementation of an abattoir survey. However all birds from a given flock will go through the same abattoir. Therefore, these results should not be interpreted as estimating the likely probability of a random bird being campylobacterpositive within one abattoir, rather it estimates random selection from the whole national flock.

Uncertainty and variability are intrinsic to most QRA models and they are often undifferentiated in their treatment within an assessment. In risk assessment uncertainty represents the degree of ignorance, and variability represents the inherent variation in the value of a particular parameter. It has been suggested that by neglecting to include such differentiation within a model could result in a final 
estimate that underestimates the uncertainty of outputs [41]. Methodological techniques which allow the discrimination and quantification of these characteristics have been reported in the literature [42, 43], but such a distinction can be difficult, making an assessment more complex and time consuming. As risk assessment techniques advance, the need for separate treatment of these model characteristics is being emphasised. Within the current model the distribution for the prevalence of positive flocks, $P_{\mathrm{fp}}$, represents uncertainty; the distribution for the withinflock prevalence of a positive flock at slaughter, $P_{\mathrm{wf} p}$, represents variability. Using the simulation method described the variability is simulated and the result is an uncertainty distribution for $P_{\mathrm{pb}}$.

In summary, this paper presents the rearing module of a QRA model to investigate human infection with campylobacter from the consumption of chicken meat/products. The aim of this module is to estimate the probability that a random bird selected from Great Britain's national flock will be campylobacterpositive at the point of slaughter. Model results indicate that $50 \%$ of randomly selected birds from the national flock will have a probability of between 0.52 and 0.54 of being campylobacter-positive. The mean value for this probability is $0 \cdot 53$. Sensitivity analysis has indicated that the time of first successful exposure is a critical control point however this is a difficult factor to control. It may be that this, in conjunction with control points identified in later stages in the supply chain will result in a significant reduction in the level of human illness. In conclusion, this communication illustrates the application of QRA modelling to microbial food safety problems and the use of such models to identify risk-mitigation strategies.

\section{ACKNOWLEDGEMENTS}

The authors thank Giles Paiba for his contribution in the preparation of this manuscript. Further the authors thank Andrew Gibson from Premier Poultry and Sheila Gray from Grampian Country Food Group for the kind provision of data. This research was funded by the Veterinary Laboratories Agency.

\section{REFERENCES}

1. Communicable Disease Surveillance Center (CDSC). CDR 1999: 9 (2).

2. Tompkins DS, Hudson MJ, Smith HR, et al. A study of infectious intestinal disease in England: microbiological findings in cases and controls. Commun Dis Publ Hlth 1999; 2: 108.

3. Saleha AA, Mead GC, Ibrahim AL. Campylobacter jejuni in poultry production and processing in relation to public health. World's Poult Sci J 1998; 54: 49-58.

4. Harris NV, Weiss NS, Nolan CM. The role of poultry and meats in the etiology of Campylobacter jejuni/coli enteritis. Am J Publ Hlth 1986; 76: 407-11.

5. Van De Giessen A, Mazurier S-I, Jacobs-Reitsma W, et al. Study on the epidemiology and control of Campylobacter jejuni in poultry broiler flocks. Appl Environ Microbiol 1992; 58: 1913-7.

6. Evans S. A longitudinal study of thermophillic campylobacter infection of poultry broiler flocks in UK. Report for Animal Health Veterinary Group, MAFF, Tolworth, 1996.

7. Jacobs-Reitsma WF, Van De Giessen AW, Bolder NM, Mulder RWAW. Epidemiology of Campylobacter spp. at two Dutch broiler farms. Epidemiol Infect 1995; 114: 413-21.

8. Covello VT, Merkhofer MW. Risk assessment methods: approaches for assessing health and environmental risks. New York: Plenum Press, 1993.

9. Whiting RC, Buchanan RL. Development of a quantitative risk assessment model for Salmonella enteritidis in pasteurized liquid eggs. Int J Food Microbiol 1997; 36: 111-25.

10. Cassin MH, Lammerding AM, Todd ECD, Ross W, McColl RS. Quantitative risk assessment for Escherichia coli $\mathrm{O} 157: \mathrm{H} 7$ in ground beef hamburgers. Int J Food Microbiol 1998; 41: 21-44.

11. Nauta MJ, Heuvelink AE. Exposure assessment of Shiga toxin producing E. coli O517 via beef and beef products in The Netherlands. Poster presentation at Society for Risk Analysis Annual Meeting, Arizona, 1998.

12. USDA. Salmonella enteritidis risk assessment. http:// www.fsis.usda.gov/ophs/risk/index.htm, 1998.

13. USDA. Escherichia coli $\mathrm{O} 157: \mathrm{H} 7$ in beef risk assessment project. http://www.fsis.usda.gov/ophs/ ecolrisk/home.htm, 1999.

14. Byrd JA, Corrier DE, Hume ME, Bailey RH, Stanker LH, Hargis BM. Incidence of campylobacter in crops of preharvest market-age broiler chickens. Poul Sci 1998; 77: 1303-5.

15. Gregory E, Barnhart H, Dreesen DW, Stern NJ, Corn JL. Epidemiological study of Campylobacter spp. in broilers: source, time of colonization, and prevalence. Avian Dis 1997; 41: 890-8.

16. Humphrey TJ, Henley A, Lanning DG. The colonization of broiler flocks with Campylobacter jejuni: some epidemiological investigations. Epidemiol Infect 1993; 110: 601-7.

17. Vose DJ Quantitative risk analysis: A guide to MonteCarlo simulation modelling. Chichester: John Wiley \& Sons Ltd., 1996.

18. Ministry of Agriculture Fisheries and Food (MAFF). Ministry of Agriculture Fisheries and Food (MAFF) statistics: Poultry and poultrymeat statistics notice, 1999. 
19. Shanker S, Lee A, Sorrell TC. Horizantal transmission of Campylobacter jejuni amongst broiler chicks: experimental studies. Epidemiol Infect 1990; 104: 101-10.

20. Bailey NTJ. The mathematical theory of infectious diseases and its applications. Great Britain: Charles Griffin and Company, 1975.

21. Fukuda K, Sugawa K, Ishii K. Simulation of infectious disease by Reed-Frost model with proportion of immune and innapparent infection. Comput Biol Med 1984; 14: 209-15.

22. Advisory Committee on the Microbiological Safety of Food. Report on poultry meat. London: HMSO, 1996.

23. McBride G, Foenander F. Territorial behaviour in flocks of domestic fowls. Nature 1962; 192: 102.

24. Collias NE, Collias EC, Hunsaker D, Minning L. Locality fixation, mobility and social organisation within an unconfined population of Red Jungle fowl. An Behav 1966; 14: 550-9.

25. McBride G, Parker IP, Foenander F. The social organisation and behaviour of the feral domestic fowl. Part 1. Field studies on North West Island. An Beh Mono 1969; 2: 127-81.

26. Wood-Gush DGM, Duncan IJH, Savory CJ. Observations on the social behaviour of domestic fowl in the wild. Biol Behav 1978; 3: 193-205.

27. Tribe A. Environmental design and site attachment in flocks of broiler chickens. M.Sc. Thesis, University of Queensland, 1980.

28. Pamment P, Foenander F, McBride G. The social and spatial organisation of male behaviour in mated domestic fowl. Appl An Ethol 1983; 9: 341-9.

29. Preston AP, Murphy LB. Movement of broiler chickens reared in commercial conditions. Bri Poul Sci 1989; 30: 519-32.

30. Annan-Prah A, Jnac M. The mode of spread of Campylobacter jejuni/coli to broiler flocks. J. Vet Med B 1988; 35: 11-8.

31. Engvall A, Bergqvist A, Sandstedt K, DanielssonTham M-L. Colonization of broilers with campylobacter in conventional broiler-chicken flocks. Acta Vet Scand 1986; 27 : 540-7.

32. Jacquez JA. A note on chain-binomial models of epidemic spread: what is wrong with the Reed-Frost formulation? Math Biosci 1987; 87: 73-82.

33. $\mathrm{Ng} \mathrm{J}$, Orav EJ. A generalized chain binomial model with application to HIV infection. Math Biosci 1990; 101: 99-119.

34. Stuart J, Sufi F, McNulty C, Park P. Outbreak of campylobacter enteritis in a residential school associated with bird pecked bottle tops. CDR 1997; 7 (Rev 3): R38-R40.

35. Stern NJ. Colonisation of poultry by Campylobacter jejuni and the potential for intervention. J Food Protect 1988; $51: 831$.

36. Shreeve JE, Toszeghy T, Pattison M, Newell DG. Sequential spread of campylobacter infection through a multi-pen broiler house. Poster presentation at 10th International Workshop on Campylobacter, Helicobacter and Related Organisms, Baltimore. 1999. 